\title{
APLICAÇÃO DA ESPECTROSCOPIA DE RESSONÂNCIA MAGNÉTICA NUCLEAR PARA ESTUDOS DE DIFUSÃO MOLECULAR EM LÍQUIDOS: A TÉCNICA DOSY
}

\author{
Alexandre Araújo de Souza* \\ Departamento de Química, Universidade Federal do Piauí, Campus da Ininga, 64049-550 Teresina - PI \\ Antonio Laverde Jr. \\ Instituto de Química, Universidade Estadual de Campinas, CP 6154, 13083-970 Campinas - SP
}

Recebido em 12/9/01; aceito em 22/2/02

\begin{abstract}
USING NUCLEAR MAGNETIC RESONANCE SPECTROSCOPY TO STUDY MOLECULAR DIFFUSION IN LIQUIDS: THE DOSY TECHNIQUE. Diffusion coefficients provide uniquely detailed and easily interpreted information on molecular organization and phase structure. They are quite sensitive to structural changes, and to binding and association phenomena, in particular for liquid colloidal or macromolecular systems. This paper describes the principles of diffusion measurements in liquids by pulsed magnetic field gradient spin-echo (PFG-SE) NMR spectroscopy. The important PFG-SE technique known as DOSY is presented and discussed. This is a noninvasive technique that can provide individual multicomponent translational diffusion coefficients with good precision in a few minutes, without the need for radioactive isotopic labelling.
\end{abstract}

Keywords: diffusion in liquids; DOSY; pulsed field gradient spin-echo NMR.

\section{A DIFUSÃO MOLECULAR}

Os coeficientes de difusão fornecem informações importantes sobre organização molecular e estrutura de fases. Há várias maneiras de medir coeficientes de difusão em líquidos, destacando-se as técnicas: de espalhamento de luz ${ }^{1}$; de cela de diafragma ${ }^{2}$; interferométrica de Gouy $^{3}$; de análise por injeção em fluxo (FIA ${ }^{4}$; de dispersão de Taylor-Aris ${ }^{5-7}$ e de ressonância magnética nuclear $(\mathrm{RMN})^{8,9}$. O presente artigo tem por objetivo apresentar os fundamentos da medida de difusão molecular por RMN, discutindo particularmente a técnica DOSY.

A difusão molecular consiste nos movimentos aleatórios (brownianos) das moléculas, devidos à energia térmica do sistema ${ }^{10}$. Verifica-se, experimentalmente, que a probabilidade $\mathrm{p}(\mathrm{x}, \mathrm{t}) \mathrm{dx}$ do centro de massa de uma molécula, inicialmente na posição $\mathrm{x}$, ter se deslocado para uma nova posição $\mathrm{x}+\mathrm{dx}$, bem próxima da inicial, após o tempo t, é dada pela equação 1 ,

$p(x, t) d x=\sqrt{\frac{1}{4 \pi D t}} \cdot \exp \left[-\frac{x^{2}}{4 D t}\right] d x$

onde D é o coeficiente de difusão da molécula. Valores típicos de coeficientes de difusão em líquidos, a temperaturas moderadas (25$30{ }^{\circ} \mathrm{C}$ ), vão desde $10^{-12} \mathrm{~m}^{2} \mathrm{~s}^{-1}$ (polímeros de alta massa molar, em solução) até $10^{-9} \mathrm{~m}^{2} \mathrm{~s}^{-1}$ (moléculas pequenas, em soluções pouco viscosas $)^{8}$.

A equação de Stokes-Einstein ${ }^{11}$ (equação 2) fornece a dependência do coeficiente de difusão de uma partícula esférica, em solução, à diluição infinita, com o seu raio hidrodinâmico r, com a viscosidade $\eta$ do solvente e com a temperatura $\mathrm{T}$ da solução ( $\mathrm{k}$ é a constante de Boltzmann). O fator 6 é usado quando a partícula for muito menor que as moléculas do solvente. Caso contrário, esse fator é substituído por um número menor ${ }^{11}$. Modificações dessa equação

*e-mail: alesouza@ufpi.br podem ser feitas para o caso de partículas não esféricas, em particular as de forma elipsoidal ${ }^{12}$.

$$
\mathrm{D}=\frac{\mathrm{kT}}{6 \pi \eta \mathrm{r}}
$$

\section{O MÉTODO DOS ECOS DE SPIN PARA MEDIR DIFUSÃO MOLECULAR}

Medidas de difusão por RMN foram feitas desde a descoberta dos ecos de spin por Hahn ${ }^{13}$, em 1950. Neste trabalho pioneiro, vários efeitos sobre os ecos de spin foram descobertos e corretamente interpretados, em especial o efeito da difusão molecular sobre as amplitudes dos ecos de spin. Basicamente, a técnica de ecos de spin monitora as amplitudes dos ecos de spin, na presença de um gradiente linear de campo magnético, para medir a difusão molecular.

Na sequiência de ecos de spin de Hahn $\left[90^{\circ}-\tau-180^{\circ}-\tau-(\text { eco)- }]_{n}\right.$, a perfeita refocalização do vetor magnetização só ocorre se cada spin nuclear (de constante magnetogírica $\gamma$ ) se mantiver sob a ação de um campo magnético homogêneo, durante o tempo $2 \tau$. Na prática, as moléculas difundem-se através de um gradiente (G) de campo magnético. Portanto, a refocalização não será perfeita e a amplitude $\mathrm{A}(2 \tau)$ do eco de spin será reduzida, de acordo com a equação 3 . Em princípio, a medida da difusão molecular pode ser feita pela sequiência de Hahn. Entretanto, é difícil separar as contribuições da relaxação transversal $\left(\mathrm{T}_{2}\right)$ e da difusão molecular (D).

$$
\frac{\mathrm{A}(2 \tau)}{\mathrm{A}(0)}=\exp \left[-\left(\frac{2 \tau}{\mathrm{T}_{2}}\right)-\frac{2}{3} \gamma^{2} \mathrm{G}^{2} \mathrm{D} \tau^{3}\right]
$$

A sequiência de Carr e Purcell ${ }^{14}, 90^{\circ}-\tau$ - [180 $\left.-\tau-(e c o)-\tau-180^{\circ}-t-(e c o)-\right]_{n}$, constitui-se em uma modificação da seqüência de Hahn, de forma que o efeito de atenuação do eco, devido à difusão molecular, pode ser separado do efeito devido a $\mathrm{T}_{2}$ (equação 4). Assim, Carr e Purcell ${ }^{14}$ puderam, em 1954 , medir para a água pura, a $25{ }^{\circ} \mathrm{C}$, o valor $\mathrm{D}=(2,5 \pm 0,3) \times 10^{-9} \mathrm{~m}^{2} \mathrm{~s}^{-1}$. 


$$
\frac{A(t)}{A(0)}=\exp \left[-\frac{t}{T_{2}}\right] \exp \left[-\frac{1}{3}(\gamma G \tau)^{2} D t\right]
$$

\section{TÉCNICAS QUE UTILIZAM GRADIENTES DE CAMPO MAGNÉTICO PULSADOS}

Na prática, a gama de valores de coeficientes de difusão que podem ser medidos, usando gradientes de campo magnético estáticos, encontra-se limitada. Por exemplo, valores elevados de gradientes devem ser utilizados para medir coeficientes de difusão pequenos, o que provoca um grande alargamento do eco obtido, com conseqüente diminuição da sua amplitude e da razão sinal-ruído, aumentando a dificuldade de detecção. Contudo, o experimento de ecos de spin foi aperfeiçoado significativamente na década de 1960 pela introdução de gradientes pulsados, solucionando os problemas decorrentes do uso de gradientes estáticos ${ }^{8,15-17}$.

A técnica de ecos de spin com gradientes de campo magnético pulsados (PFGSE) consiste em uma sequiência do tipo $90^{\circ}-\tau-180^{\circ}$, onde se aplicam dois pulsos de gradiente: um antes e outro após o pulso RF de $180^{\circ}$ (Figura 1). A intensidade g do pulso de gradiente deve ser muito superior ao gradiente estático $g_{0}$, proveniente da nãohomogeneidade do campo magnético $\left(\mathrm{g}>>\mathrm{g}_{0}\right)$. A duração $\delta$ do pulso de gradiente deve ser curta quando comparada com o tempo t entre os pulsos $\mathrm{RF}(\delta<<\mathrm{t})$. Assim, a amplitude do eco será dada pela equação 5 ,

$$
\frac{A(2 \tau)}{A(0)}=\exp \left[-\frac{2 \tau}{T_{2}}\right] \exp \left[-D(\operatorname{yg} \delta)^{2}\left(\Delta-\frac{\delta}{3}\right)\right]
$$

onde $\Delta$ é o intervalo de tempo entre os inícios dos dois pulsos de gradiente consecutivos (Figura 2). O termo de correção $-\delta / 3$ é uma conseqüência da forma retangular dos pulsos de gradiente. As vantagens desta técnica, em relação às que utilizam gradientes estáticos, são duas: (a) o efeito de atenuação do eco, devido à difusão, pode

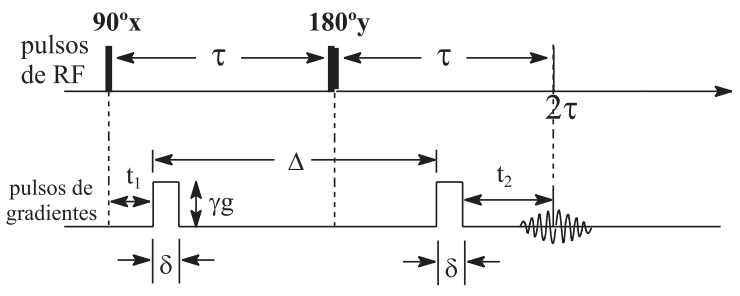

Figura 1. Seqüencia de pulsos para o experimento de ecos de spin com gradientes de campo magnético pulsados (PFGSE)

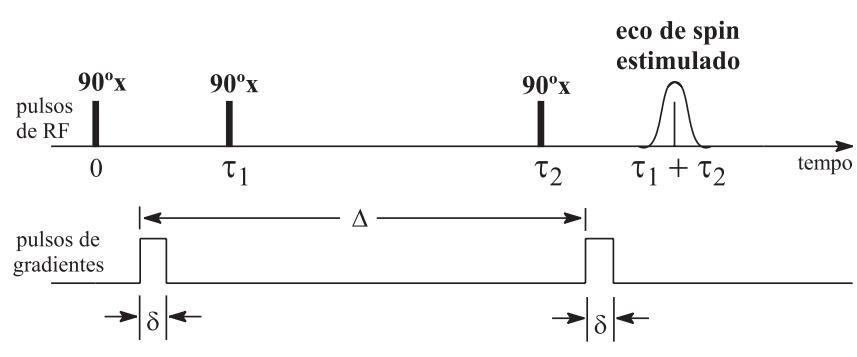

Figura 2. Seqüência de pulsos para o experimento de ecos de spin estimulados ser separado do efeito devido à relaxação transversal $\mathrm{T}_{2}$, conduzindo-se o experimento em um intervalo $\tau$ fixo, entre os pulsos RF e variando-se a chamada área do pulso de gradiente: $\mathrm{q}=\gamma \mathrm{g} \delta$; (b) a detecção do eco de spin é feita sob um campo magnético homogêneo ${ }^{18,19}$.

Uma variação importante da técnica PFGSE é a chamada sequiência de ecos de spin estimulados ${ }^{8,20,21}$. Esta técnica consiste em uma seqüência de três pulsos de RF de $90^{\circ}$ (Figura 2), onde é aplicado um pulso de gradiente entre os dois primeiros pulsos de RF e um segundo pulso de gradiente após o terceiro pulso RF. Anteriormente, Hahn ${ }^{13}$ já discutia o conceito de ecos estimulados e a sua utilização para medidas de difusão. Uma seqüência de três pulsos de RF de $90^{\circ}$ pode resultar em até cinco ecos de spin. $O$ conceito importante desta técnica, para as medidas de difusão, é que a atenuação, pela difusão, do primeiro eco - chamado de eco estimulado - compete tanto com a relaxação transversal $T_{2}$, quanto com a relaxação longitudinal $T_{1}$. Quando ocorre troca química, por exemplo, $\mathrm{T}_{2}$ pode ser bem menor que $\mathrm{T}_{1}$ e pode ser mais vantajoso usar a técnica baseada em ecos estimulados. A atenuação do eco estimulado é dada pela equação 6 ,

$\frac{A(T+2 \tau)}{A(0)}=\frac{1}{2} \cdot \exp \left[-\left(\frac{2 \tau}{T_{2}}\right)-\left(\frac{T}{T_{1}}\right)\right] \exp \left[-D(\gamma g \delta)^{2}\left(\Delta-\frac{\delta}{3}\right)\right]$

onde T é o tempo entre o segundo e o terceiro pulso RF de $90^{\circ}$. Da mesma forma que antes, mantendo-se $\tau$ e T constantes, consegue-se separar os efeitos de $\mathrm{T}_{1}$ e $\mathrm{T}_{2}$ do efeito da difusão, na atenuação do eco.

\section{A TÉCNICA DOSY}

Em 1992, Morris e Johnson ${ }^{22}$ desenvolveram uma técnica analítica não-invasiva, baseada na metodologia dos ecos de spin com gradientes de campo magnético pulsados (PFGSE), que permite discriminar e caracterizar os diversos componentes de uma mistura. Esta técnica foi denominada DOSY ("Diffusion Ordered Spectroscopy"). Em um espectro DOSY, os deslocamentos químicos são apresentados em uma dimensão e os coeficientes de difusão moleculares são mostrados na outra (Figura 3). Esta técnica pode detectar qualquer substância que apresente núcleos ativos em $\mathrm{RMN}^{23}$. Para moléculas

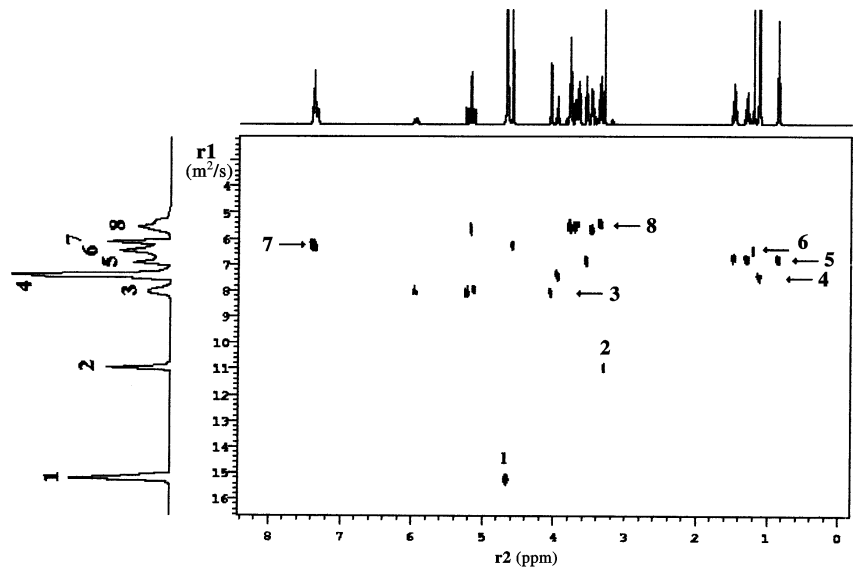

Figura 3. Espectro de DOSY $\left({ }^{1} \mathrm{H}, 500 \mathrm{MHz}\right.$, CGSTESL, $\left.30{ }^{\circ} \mathrm{C}\right)$ de mistura de álcoois ( $2=$ metanol; 3 =álcool alílico; 4 = isopropanol; $5=n$-butanol; $6=$ t-butanol; 7 = álcool benzílico $)$ e sacarose $(=8)$, em água deuterada $(1$ = HOD). Projeção do "espectro de difusão" $\left(\mathrm{m}^{2} / \mathrm{s}\right)$ em F1 e projeção do espectro de $R M N$ de ${ }^{I} \mathrm{H}$ (ppm) em F2 
orgânicas, normalmente monitora-se o núcleo de ${ }^{1} \mathrm{H}$. Em virtude de sua habilidade em resolver misturas complexas e permitir a simultânea identificação dos compostos, a técnica DOSY vem sendo sugestivamente denominada pelos químicos como a "cromatografia de spins" 24 .

Considere uma mistura com n componentes. Durante um experimento DOSY, varia-se a área $(\mathrm{q}=\gamma \mathrm{g} \delta)$ do gradiente de campo magnético pulsado e obtem-se espectros de RMN para cada valor de q. Na Figura 4, pode ser visto o decaimento exponencial através das intensidades $\mathrm{I}(\mathrm{q}, v)$ dos sinais de RMN, em função da área do gradiente. Este decaimento é descrito pela equação 7 ,

$$
\mathrm{I}(\mathrm{q}, v)=\sum_{\mathrm{n}}\left[\mathrm{A}_{\mathrm{n}}(v) \cdot \exp \left[-\mathrm{D}_{\mathrm{n}}\left(\Delta-\frac{\delta}{3}\right) \mathrm{q}^{2}\right]\right]
$$

onde $A_{n}(v)$ é a intensidade dos sinais de $R M N$ para $q=0 ; D_{n}$ é o coeficiente de difusão de cada componente e $\Delta$ é o tempo durante o qual a difusão está sendo monitorada. Após a transformada de Fourier é aplicada uma transformada inversa de Laplace ${ }^{25}$, gerando-se o espectro DOSY (Figura 3).

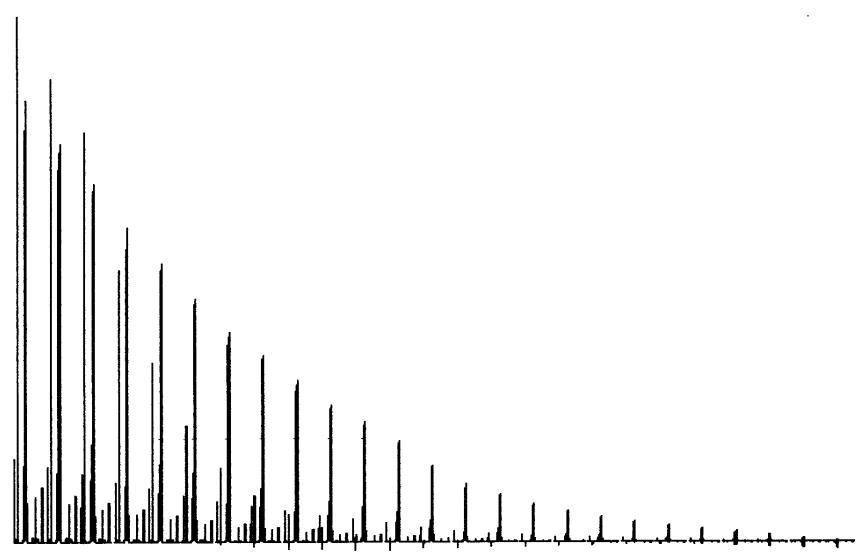

Figura 4. Série de 25 espectros de RMN de ${ }^{l} \mathrm{H}(500 \mathrm{MHz})$ obtidos variando a intensidade $(\mathrm{g})$ dos gradientes de campo pulsados. A intensidade dos sinais decai exponencialemte (Eq. 7) em função da área $(q=\gamma g \delta)$ do gradiente

As limitações da técnica DOSY são aquelas inerentes à RMN: por exemplo, a baixa sensibilidade. Se houver superposição de sinais de RMN de diferentes componentes e se os respectivos coeficientes de difusão diferirem por um fator menor do que 2, um único sinal aparecerá na dimensão da difusão do espectro de DOSY, correspondendo ao coeficiente de difusão médio ${ }^{25,26}$. Uma solução para este problema seria a utilização de versões tridimensionais de DOSY. Outra limitação desta técnica é a necessidade de se dispor de um espectrômetro de RMN com gradientes de campo magnético pulsados.

Uma grande vantagem da utilização de um experimento rápido e acurado como DOSY é o fato de o mesmo proporcionar uma visão global da dinâmica translacional dos constituintes de uma mistura, sejam eles moléculas pequenas, macromoléculas, complexos ou agregados moleculares. Outras vantagens importantes são listadas abai$\mathrm{XO}^{27}$ :

- as impurezas podem ser detectadas sem nenhuma interferência;

- espécies de interesse podem ser selecionadas para análise;

- componentes concentrados podem ser acompanhados sem dificuldade;
- a presença de espécies em equilíbrio pode ser avaliada e quantificada.

Há dois fatores essenciais para o bom desenvolvimento da técnica de DOSY28.

- procedimentos e tecnologia capazes de codificar a informação sobre o movimento translacional de uma vasta extensão de substâncias dentro de um conjunto de dados de RMN e

- a transformação e visualização dos dados codificados de RMN para a dimensão da difusão.

O primeiro fator vem sendo alcançado com o desenvolvimento contínuo de sequiências de pulsos de gradiente de campo visando principalmente a resolução dos deslocamentos químicos e a estabilização do sinal ${ }^{29}$. A estabilidade do sinal é crucial em DOSY e um critério importante para o sucesso da mesma é minimizar as perturbações, tais como as correntes de vórtice ("eddy currents") induzidas na amostra, o acoplamento entre a bobina de gradiente e o campo magnético principal, e os distúrbios do sistema de trava da frequiência e do campo, que combinados levam à degradação da forma do sinal de $\mathrm{RMN}^{18,19}$. Uma forma engenhosa de corrigir o problema de correntes de vórtice consiste em aplicar pulsos de gradientes com polaridades invertidas, alternadamente, pois um pulso compensa o efeito do outro. Várias sequiências de pulsos de RMN foram desenvolvidas no intuito de reduzir ao máximo alguns destes e outros efei$\operatorname{tos}^{29}$. Entretanto, a eficiência de uma ou outra sequiência depende do tipo de amostra a ser analisada e, sob determinadas circunstâncias, o emprego de uma prevalecerá sobre a outra. Assim, baseado na tecnologia atual, Johnson ${ }^{18}$ resumiu as melhores situações para o emprego das seqüências de DOSY mais importantes (Figura 5). $\mathrm{Na}$ ausência de efeitos de troca química ou acoplamento de spins e quando pequenos gradientes são requeridos, as seqüências BPPSTE ${ }^{26}$ ("Bipolar Pulse Pairs Stimulated Echo") e GCSTE"29 ("Gradient Compensated Stimulated Echo") são escolhas razoáveis. Quando os efeitos de troca não estão presentes e gradientes modestos bastam para a aquisição, a melhor escolha é a sequiência GCSTESL ${ }^{29}$ (“Gradient Compensated Stimulated Echo Spin Lock"). Nas situações que requerem refocalização do deslocamento químico, com-

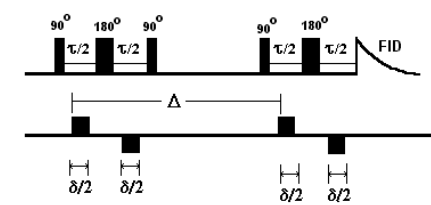

BPPSTE

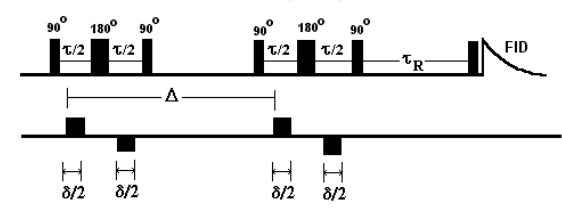

BPPLED
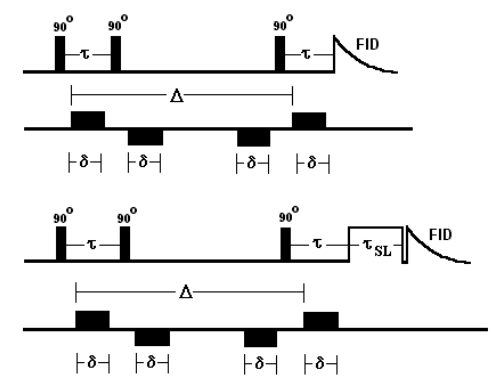

GCSTESL

Figura 5. Algumas das principais seqüências de pulsos utilizadas nos experimentos de DOSY (adaptado da Ref. 29) 
pensação de gradientes básicos e gradientes fortes, a sequiência BPPLED 30 ("Bipolar Pulse Pairs Longitudinal Eddy currents Delay") seria a mais indicada. Dessa forma, a escolha de uma seqüência de pulsos para DOSY depende basicamente da capacidade da instrumentação disponível e da natureza do sistema sob estudo.

O segundo fator é considerado o coração do experimento. Para a transformação e visualização dos sinais são necessários programas computacionais eficientes com algoritmos capazes de analisar os conjuntos de dados resultantes das transformadas de Fourier e de Laplace inversa. O maior desafio é a conversão dos decaimentos das intensidades dos sinais de RMN obtidos pela transformada de Fourier, aplicando a transformada de Laplace inversa. Programas como CONTIN, DISCRETE, SPLMOD e MaxEnt têm sido empregados com sucesso ${ }^{18,19}$. Entretanto, é recomendado que os usuários destes programas estejam atentos ao surgimento de artefatos e imprevistos. Com relação ao processamento dos espectros, um cuidado especial deve ser tomado com a linha base. A correção da mesma minimiza erros padrões obtidos, assegurando a exatidão dos coeficientes de difusão adquiridos. Se a correção da fase não for utilizada, o efeito cumulativo de sobreposição de linhas distorcerá todas as alturas dos picos, comprometendo conseqüentemente a precisão da análise ${ }^{29}$.

\section{DOSY Tridimensional}

Além da introdução de novas seqüências de pulsos, as quais têm proporcionado o aumento da resolução do DOSY, uma série de versões tridimensionais deste experimento têm sido desenvolvidas recentemente, com o mesmo objetivo. Nos experimentos de DOSY $3 \mathrm{D}$, a coordenada de difusão está associada a um experimento de RMN 2D convencional, onde as matrizes tridimensionais geradas apresentam duas dimensões de deslocamentos químicos e uma de coeficientes de difusão. Algumas destas versões podem ser conferidas: DOSY-COSY ${ }^{31}$, DOSY-NOESY ${ }^{32}$, DOSY-TOCSY ${ }^{33}$ e DOSY$\mathrm{HMQC}^{34}$. Todos estes experimentos tridimensionais foram desenvolvidos para diminuir a sobreposição espectral de sinais e facilitar a identificação de compostos em misturas. As limitações mais significantes dos mesmos são o longo tempo de aquisição e a necessidade de muita memória para armazenagem dos dados.

Enfim, há uma riqueza de informações físico-químicas disponíveis em experimentos de PFGSE e a principal proposta de um método como DOSY é estender as aplicações para sistemas complexos.

\section{APLICAÇÕES}

Além da importância do conhecimento dos coeficientes de difusão para a descrição de qualquer processo que envolva fluxo de massa, estes parâmetros fornecem informações importantes sobre vários processos que ocorrem em solução, os quais podem ser facilmente avaliados por DOSY. A aplicabilidade desta metodologia na análise de misturas tem sido demonstrada em um número de sistemas complexos, incluindo misturas de moléculas orgânicas pequenas, extratos de tecidos e biofluidos ${ }^{18,19}$.

Uma das aplicações mais comuns é a análise de misturas visando a verificação da distribuição das espécies no meio de acordo com suas massas moleculares. Um exemplo clássico é o trabalho de Morris e colaboradores ${ }^{25}$, o qual mostra claramente a correlação existente entre massa molecular e coeficiente de difusão, através da análise de uma mistura de álcoois em solução aquosa. Um exemplo similar pode ser visto na Figura 3. Uma série de trabalhos com polímeros também tem ilustrado muito bem esta aplicação ${ }^{35,36}$. No trabalho de Young e colaboradores ${ }^{35}$, com polímeros dendriméricos, de arquitetura interna idêntica, mas com grupos funcionais terminais ácidos, neutros e básicos, as análises de DOSY revelaram que alguns dos polímeros avaliados aumentavam ou reduziam de tamanho, em resposta à mudança de pH, devido à dependência do raio hidrodinâmico em relação ao $\mathrm{pH}$. Outros exemplos da praticidade de DOSY podem ser avaliados através de dois trabalhos mais recentes ${ }^{37,38}$, os quais mostram a análise de misturas complexas e heterogêneas de frações de petróleo ${ }^{37}\left({ }^{1} \mathrm{He} \mathrm{e}^{13} \mathrm{C}\right.$-DOSY) e de açúcares de mesma massa molecular ${ }^{38}$.

Uma das aplicações mais comuns dos estudos de difusão envolve a quantificação de partição ou interação de solutos com compostos macromoleculares como ciclodextrinas ${ }^{39-41}$, micelas ${ }^{28,29,42}$, vesículas $^{22,23,43}$ e proteínas ${ }^{30,44}$. Quando um soluto difunde e, simultaneamente, interage com um destes sistemas, assumindo que a interação seja reversível, e mais rápida que a dispersão molecular, o coeficiente de difusão observado $\left(\mathrm{D}_{\text {obs }}\right)$ é uma composição do coeficiente de difusão do soluto livre $\left(\mathrm{D}_{\text {livre }}\right)$ e incorporado $\left(\mathrm{D}_{\text {compl }}\right)$ e a fração de soluto complexada $\left(f_{\text {compl }}\right)$ pode ser calculada $\left(D_{\text {obs }}=f_{\text {live }} D_{\text {live }}\right.$ $+\mathrm{f}_{\text {compl }} \mathrm{D}_{\text {compl }}$ ). Um exemplo que ilustra bem o comportamento de solutos na presença de macromoléculas hospedeiras é dado pela difusão da cicloalcanona 1 , na presença e ausência de $\beta$-ciclodextrina (Figura 6) ${ }^{41,45}$. A partir de coeficientes de difusão experimentais, podese observar que a razão de difusão era muito sensível aos fenômenos
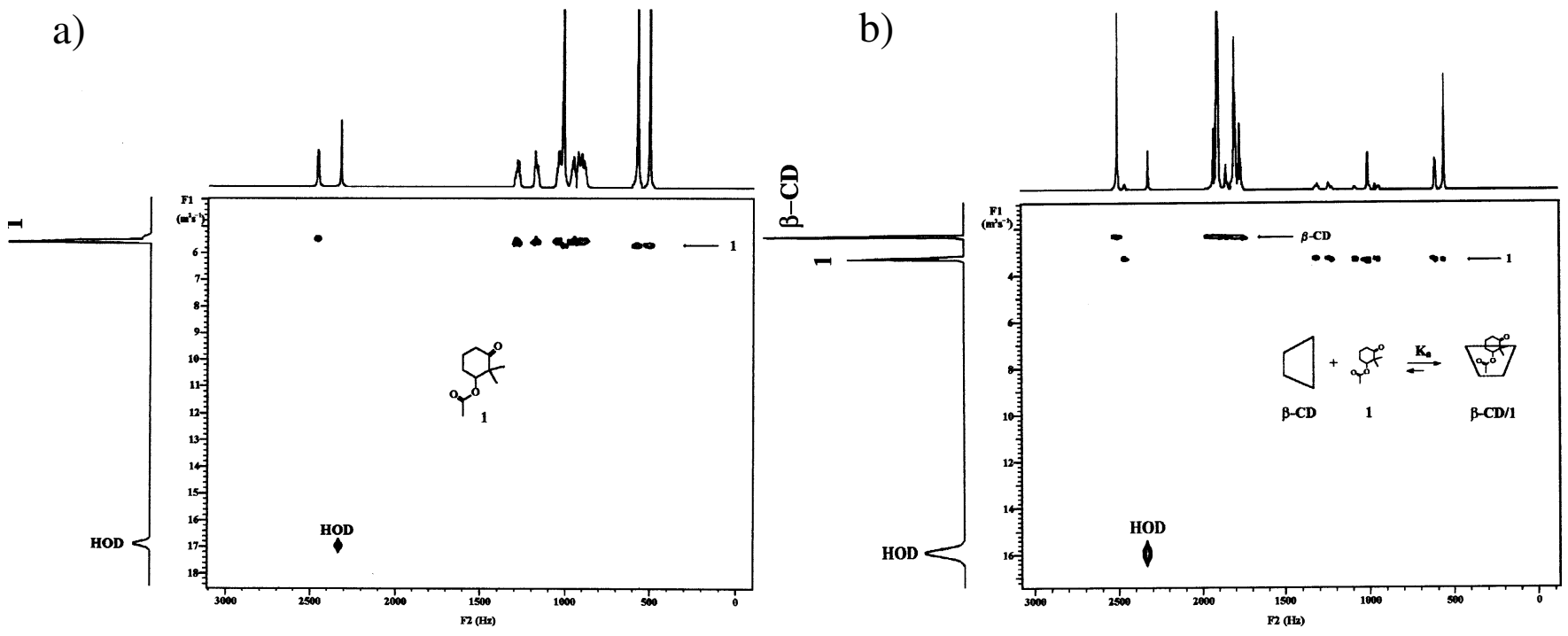

Figura 6. Espectros de DOSY $\left({ }^{1} \mathrm{H}, 500 \mathrm{Mhz}\right.$, CGSTESL, $\left.30^{\circ} \mathrm{C}\right)$ da cicloalcanona 1 em solução aquosa (15mM): (a) na ausência de ciclodextrina; (b) na presença de $\beta$-ciclodextrina $(15 \mathrm{mM})$ 
de associação, onde a diminuição do coeficiente de difusão é uma evidência clara da incorporação do soluto pelo hospedeiro. A razão de complexação entre as espécies (soluto-ciclodextrina) e a constante de associação aparente $\left(\mathrm{K}_{\mathrm{ap}}\right)$ foram calculadas e serviram de parâmetro para avaliar o grau de complexação entre os adutos.

Utilizando-se a equação de Stokes-Einstein (equação 2), podese determinar o tamanho de sistemas macromoleculares como micelas e vesículas. Neste sentido, Hinton e Johnson ${ }^{23,43}$ desenvolveram alguns estudos para caracterizar vesículas fosfolipídicas unilamelares grandes. Através dos coeficientes de difusão obtidos por DOSY, os autores calcularam os diâmetros médios das vesículas. Os resultados foram consistentes com aqueles encontrados por microscopia eletrônica e medidas por espalhamento de luz.

$\mathrm{O}$ amplo espectro de aplicabilidade desta metodologia pode ser ilustrado ainda por outros trabalhos recentes. Tillet e colaboradores $^{44}$, por exemplo, mostraram que coeficientes de difusão podem ser sensíveis a efeitos sutis, resultantes de mudança conformacional e hidratação de proteínas, fato que os têm levado a trabalhar intensivamente na investigação de mudanças conformacionais de proteínas utilizando medidas de difusão. Aplicando ${ }^{1} \mathrm{H}-\mathrm{e}{ }^{7} \mathrm{Li}$-DOSY, Keresztes e Williard ${ }^{46}$ mostraram que, em soluções de THF, o n-butil-lítio existe em equilíbrio na forma de agregados diméricos e tetraméricos, ambos tetrassolvatados. E finalmente, Kapur e colaboradores ${ }^{47}$ têm mostrado a viabilidade da utilização de ${ }^{1} \mathrm{H}-\mathrm{e}^{31} \mathrm{P}-\mathrm{DOSY}$ como sonda para entender qualitativamente a força relativa das ligações de hidrogênio entre vários componentes em uma mistura e um ligante em soluções não aquosas.

\section{AGRADECIMENTOS}

Os autores agradecem aos professores A. J. Marsaioli e F. Y. Fujiwara, do IQ/UNICAMP, pelo apoio e pelas discussões muito proveitosas sobre a técnica DOSY e suas aplicações, bem como ao CNPq, CAPES/PICDT e FAPESP pelo apoio financeiro.

\section{REFERÊNCIAS}

1. Tyrrell, H. J. V.; Harris, K. R.; Diffusion in Liquids, Butterworths: London, 1984.

2. Easteal, A. J.; Can. J. Chem. 1990, 68, 1611.

3. Paduano, L.; Sartorio, R.; Vitagliano, V.; Constantino, L.; J. Solution Chem. 1990, 19, 31.

4. Zou, G.; Liu, Z.; Wang, C.; Anal. Chim. Acta 1997, 350, 359.

5. Taylor, G. I.; Proc. Royal Soc. London Ser. A 1953, 219, 186 e 1954, 223, 446.

6. Aris, R.; Proc. Royal Soc. London Ser. A 1956, $235,67$.

7. Loh, W.; Quim. Nova 1997, 20, 541.

8. Stilbs, P.; Prog. Nucl. Magn. Reson. Spectrosc. 1987, 19, 1.

9. Gil, V. M. S.; Geraldes, C. F. G. C.; Ressonância Magnética Nuclear Fundamentos, Métodos e Aplicações, Fundação Calouste Gulbenkian: Lisboa, 1987, p. 549.
10. Jost, W. Em Diffusion in Solids, Liquids and Gases; Loebl, E. M., ed.; Academic Press: New York, 1960.

11. Levine, I.; Physical Chemistry, $3^{\text {rd }}$ ed., McGraw-Hill: New York, 1988, p. 486.

12. Edward, J. T.; J. Chem. Educ. 1970, 47, 261.

13. Hahn, E. L.; Phys. Rev. 1950, 80, 580.

14. Carr, H. Y.; Purcell, E. M.; Phys. Rev. 1954, 94, 630.

15. McCall, D. W. ; Douglas, D. C. ; Anderson, E. W.; Ber. Bunsenges. Phys. Chem. 1963, 67, 366.

16. Stejskal, E. O.; Tanner, J. E.; J. Chem. Phys. 1965, 42, 288.

17. Stejskal, E. O.; J. Chem. Phys. 1965, 43, 3597.

18. Johnson Jr., C. S.; Prog. Nucl. Magn. Reson. Spectrosc. 1999, 34, 203.

19. Johnson, C. S. Jr. Em Encyclopedia of NMR; Grant, D.M.; Harris, R.K., eds.; John Wiley \& Sons: New York, 1996, vol. 3, p. 1626.

20. Woessner, D. E.; J. Chem. Phys. 1961, 34, 2057.

21. Tanner, J. E.; J. Chem. Phys. 1970, 52, 2523.

22. Morris, K. F.; Johnson Jr., C. S.; J. Am. Chem. Soc. 1992, 114, 3139.

23. Hinton, D. P.; Johnson, C. S. Jr.; Chem. Phys. Lipids 1994, 69, 175.

24. Gounarides, J. S.; Chen, A.; Shapiro, M. J.; J. Chromatogr., B 1999, 725, 79.

25. Morris, K. F.; Stilbs, P.; Johnson Jr., C. S.; Anal. Chem. 1994, 66, 211.

26. Wu, D.; Chen, A.; Johnson, C. S. Jr.; J. Magn. Reson. Ser. A 1995, 115, 123.

27. Chen, A.; Wu, D.; Johnson, C. S. Jr.; J. Phys. Chem. 1995, 99, 828.

28. Morris, K. F.; Johnson, C. S. Jr.; J. Am. Chem. Soc. 1993, 115, 4291.

29. Pelta, M. D.; Barjat, H.; Morris, G. A.; Davis; A. L.; Hammond, S. J.; Magn. Reson. Chem. 1998, 36, 706.

30. Wu, D.; Chen, A.; Johnson, C. S., Jr.; J. Magn. Reson. Ser. A 1995, 115, 260

31. Wu, D.; Chen, A.; Johnson, C. S., Jr.; J. Magn. Reson. Ser. A 1996, 121, 88 .

32. Gozansky, E. K.; Gorenstein, D. G.; J. Magn. Reson. Ser. B 1996, 111, 94.

33. Birlirakis, N.; Guittet, E.; J. Am. Chem. Soc. 1996, 118, 13083.

34. Barjat, H.; Morris, G. A.; Swanson, A. G.; J. Magn. Reson. 1998, 131, 131.

35. Young, K. J.; Backer, G. R.; Newkome, G. R.; Morris, K. F.; Johnson, C. S. Jr.; Macromolecules 1994, 27, 3464.

36. Morris, K. F.; Johnson, C. S. Jr.; Wong, T. C.; J. Phys. Chem. 1994, 98, 603; Wu, D.; Johnson, C. S. Jr.; J. Am. Chem. Soc. 1995, 117, 7965; Gorkon, L. C. M.; Hancewicz, T. M.; J. Magn. Reson. 1998, 130, 125; Chen, A.; Jerschow, A.; Müller, N.; Macromolecules 1998, 31, 6573; Jayawickrama, D. A.; Larive, C. K.; McCord, E. F.; Roe, D. C.; Magn. Reson. Chem. 1998, 36, 755.

37. Kapur, G. S.; Findeisen, M.; Berger, S.; Fuel 2000, 79, 1347.

38. Díaz, M. D.; Berger, S.; Carbohydr. Res. 2000, 329, 1.

39. Borges, R. B.; Laverde, A. Jr.; Porto, A. L. M.; Marsaioli, A. J.; Spectrosc. Int. J. 2000, 14, 203.

40. Lin, M.; Jayawickrama, D. A.; Rose, R. A.; DelViscio, J. A.; Larive, C. K.; Anal. Chim. Acta 1995, 307, 449; Skinner, P. J.; Blair, S.; Kataky, R.; Parker, D.; New J. Chem. 2000, 24, 265; Sánchez, M.; Parella, T.; Cervelló, E.; Jaime, C.; Virgili, A.; Magn. Reson. Chem. 2000, 38, 925.

41. Laverde, A. Jr.; Conceição, G. J. A.; Queiroz, S. C. N.; Fujiwara, F. Y; Marsaioli, A. J.; Magn. Reson. Chem., submetido.

42. de Souza, A. A.; Tese de Doutorado, Universidade Estadual de Campinas, Brasil, 2001.

43. Hinton, D. P.; Johnson, C. S. Jr.; J. Phys. Chem. 1993, 97, 9064

44. Tillett, M. L.; Lian, L.-Y.; Norwood, T. J.; J. Magn. Reson. 1998, 133, 379.

45. Laverde, A. Jr.; Tese de Doutorado, Universidade Estadual de Campinas, Brasil, 2001.

46. Keresztes, I.; Williard, P. G.; J. Am. Chem. Soc. 2000, 122, 10228.

47. Kapur, G. S.; Cabrita, E. J.; Berger, S.; Tetrahedron Lett. 2000, 41, 7181. 